\title{
Introducing Type-2 Fuzzy Sets for Image Texture Modelling
}

\author{
J. Chamorro-Martínez P. Martínez-Jiménez D. Sánchez \\ Department of Computer Science and Artificial Intelligence, University of Granada \\ C/ Periodista Daniel Saucedo Aranda s/n, 18071 Granada, Spain \\ \{jesus,pedromartinez,daniel $\} @$ decsai.ugr.es
}

\begin{abstract}
In this paper, the texture property "coarseness" is modeled by means of type-2 fuzzy sets, relating representative coarseness measures (our reference set) with the human perception of this texture property. The type-2 approach allows to face both the imprecision in the interpretation of the measure value and the uncertainty about the coarseness degree associated to a measure value. In our study, a wide variety of measures is analyzed, and assessments about coarseness perception are collected from pools. This information is used to obtain type-2 fuzzy sets where the secondary fuzzy sets are modelled by means of triangular membership functions fitted to the collected data.
\end{abstract}

\section{Introduction}

For analyzing an image several kind of features can be used. From all of them, texture is one of the most popular and, in addition, one of the most difficult to characterize due to its imprecision. For describing texture, humans use vague textural properties like coarseness-fineness, orientation or regularity $[1,2]$. Among all, the coarseness-fineness is the most common one, being usual to associate the presence of fineness with the presence of texture. In this framework, a fine texture corresponds to small texture primitives (e.g. the image in Fig. 1(A)), whereas a coarse texture corresponds to bigger primitives (e.g. the image in Fig. 1(I)).

There are many measures in the literature that, given an image, capture the fineness (or coarseness) presence in the sense that the greater the value given by the measure, the greater the perception of texture [3]. However, given a measure value, there is not an immediate way to decide whether there is a fine texture, a coarse texture or something intermediate; in other words, there is not a textural interpretation.

To face this problem, fuzzy logic has been recently used for representing the textural imprecision (most of them type-1 fuzzy approaches). In many of these proposals, fuzzy logic is applied just during the process, being the output a crisp result [4]. Interesting type-1 approaches emerge from the content-based image retrieval field, where semantic data are managed by means of fuzzy sets [5]. However, these fuzzy sets are not obtained by considering the relationship between the feature and the human perception of texture. About type-2 fuzzy approaches, there are some proposals in the literature that use interval-valued fuzzy set for image restoration [6], edge detection [7] or segmentation $[8,9]$; nevertheless, to our knowledge, type-2 fuzzy sets have not been used for texture modeling.

Recent type- 1 approaches try to represent the texture and its semantic by means of fuzzy sets defined on the domain of a given texture measure [10]. A good starting point in the previous approaches in order to obtain a fuzzy model is to collect user assessments for a certain set of images where the different fulfilment degrees of the feature is well represented. However, it is almost impossible to find a clear functional correspondence between values of measures of texture features and fulfilment degrees of the corresponding fuzzy sets, because different images having the same value of a given measure are usually given different membership degrees by the users. In this situation, as it is well known, type-2 fuzzy sets is the most suitable tool to represent the uncertainty about the actual membership degree for every value in the reference set (domain of the measure).

In this paper, the texture property coarseness is modelled by means of type- 2 fuzzy sets defined on the domain of a given measure. With this approach two problems in the texture modeling will be faced: firstly, the imprecision in the interpretation of the measure value, secondly, the uncertainty about the association between measure values and degree of coarseness-fineness. For defining the fuzzy sets, information about human perception of coarseness will be used to relate the measures values with this perception.

The rest of the paper is organized as follows. In section 2 we present our methodology to obtain type- 2 fuzzy sets modelling coarseness. Results are shown in section 3, and the conclusions and future work are sumarized in section 4 . 

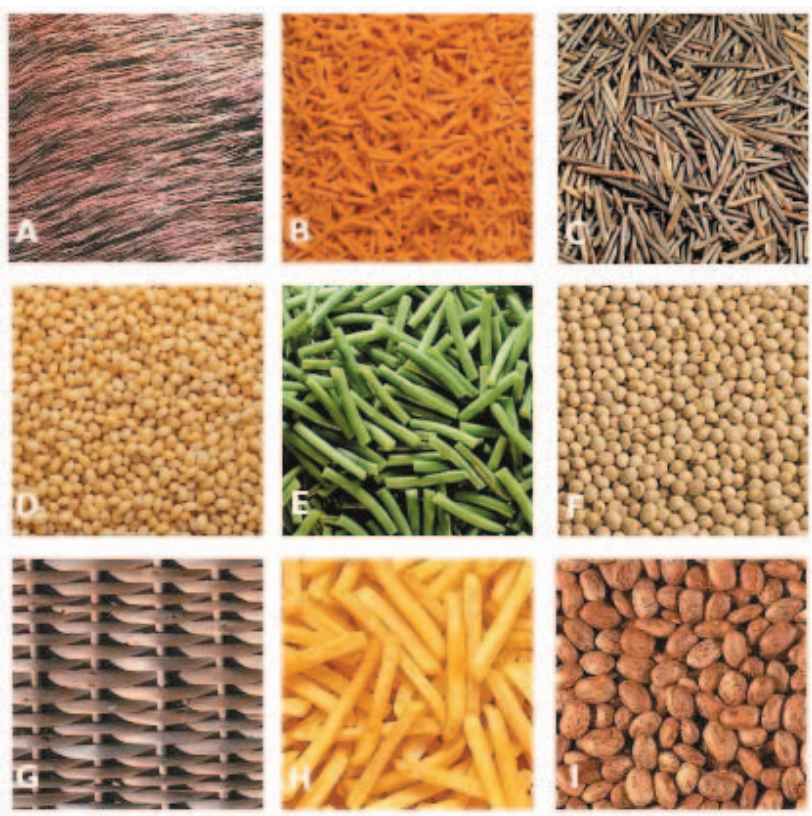

\section{Figure 1. Some examples of images with different degrees of fineness}

\section{Coarseness Type-2 Fuzzy Modeling}

As it was pointed out, there is not a clear perceptual interpretation of the value given by a coarseness-fineness measure ${ }^{1}$. In addition, there is uncertainty about the association between measure values and degree of coarseness-fineness. In order to face this problem, we propose to model the coarseness-fineness perception as a type-2 fuzzy set defined on the domain of a given measure. Let $\mathcal{P}=\left\{P_{1}, \ldots, P_{K}\right\}$ be a set of measures of coarseness-fineness and let $\tilde{\mathcal{T}}_{k}$ be a type- 2 fuzzy set defined on the domain of $P_{k} \in \mathcal{P}$ representing the concept of "coarseness-fineness". Thus, the type-2 membership function ${ }^{2}$ associated to $\tilde{\mathcal{T}}_{k}$ will be defined as

$$
\tilde{\mathcal{T}}_{k}: \mathbb{R} \times[0,1] \rightarrow[0,1]
$$

where $\tilde{\mathcal{T}}_{k}(x, u)$ represents the degree to which $u$ is in the fuzzy set representing the membership degree of $x$ in $\tilde{\mathcal{T}}_{k}$. The secondary fuzzy set representing the membership of every element $x \in \mathbb{R}$ to the type-2 fuzzy set $\tilde{\mathcal{T}}_{k}$ is denoted by $\tilde{\mathcal{T}}_{k, x}$, with membership function $\tilde{\mathcal{T}}_{k, x}:[0,1] \rightarrow[0,1]$.

Given a measure $P_{k} \in \mathcal{P}$, we propose to obtain $\tilde{\mathcal{T}}_{k}$ by finding a type- 2 membership function defined on $P_{k}$

\footnotetext{
${ }^{1}$ Let us remark that "coarseness" and "fineness" are opposite, and hence related, properties

${ }^{2}$ From now on, in order to simplify the notation, we will use the same notation $\tilde{\mathcal{T}}_{k}$ for the type-2 fuzzy set and for the type- 2 membership function that defines it
}

modeling the perception degree of coarseness-fineness. To do it, we will use a set $\mathcal{I}=\left\{I_{1}, \ldots, I_{N}\right\}$ of $N$ images that fully represent the different degrees of fineness. Thus, for each image $I_{i} \in \mathcal{I}$, we will obtain (a) a human assessment of the fineness degree perceived, noted as $v^{i}$, which will be collected by means of a poll with human subjects (section 2.1), and (b) a value calculated applying the measure $P_{k} \in \mathcal{P}$ to the image $I_{i}$, noted as $m_{k}^{i}$. From the multiset $\Psi_{k}=\left\{\left(m_{k}^{1}, v^{1}\right), \ldots,\left(m_{k}^{N}, v^{N}\right)\right\}$, the function $\tilde{\mathcal{T}}_{k}$ will be estimated (section 2.2$)$.

\subsection{Assessment collection}

In this section, the way to obtain the set $\Gamma=$ $\left\{v^{1}, \ldots, v^{N}\right\}$ of assessments associated to $\mathcal{I}$ will be described.

\subsubsection{The texture image set}

A set $\mathcal{I}=\left\{I_{1}, \ldots, I_{N}\right\}$ of $N=80$ images representative of the concept of coarseness-fineness has been selected. Fig. 1 shows some images extracted from the set $\mathcal{I}$. Such set has been selected satisfying the following properties: (1) it covers the different presence degrees of fineness, (2) the number of images for each presence degree is representative enough, and (3) each image shows, as far as possible, just one presence degree of fineness. Due to the third property, each image can be viewed as "homogeneous" with respect to the fineness degree represented, i.e., if we select two random windows (with a dimension which does not "break" the original texture primitives and structure), the perceived fineness will be the same for each window (and also respect to the original image).

As we explained, given an image $I_{i} \in \mathcal{I}$, a set of measures $\mathcal{P}$ will be applied on it. In fact, and thanks to the third property, we really can apply these measures to subimages (windows), assuming that the human assessment associated to that subimage will be the human assessment associated to the whole image. From now on, we will note as $m_{k}^{i, w}$ the result of applying the measure $P_{k} \in \mathcal{P}$ to the $w$-th window of the image $I_{i}$. Therefore, the multiset $\Psi_{k}$ is redefined as $\Psi_{k}=\left\{\left(m_{k}^{i, w}, v^{i}\right), i=1, \ldots, N ; w=\right.$ $1, \ldots, W\}$, with $N$ being the number of images and $W$ the number of windows considered for each image. From now on, we will note as $\mathbf{p}_{k}^{i, w}$ the pair $\left(m_{k}^{i, w}, v^{i}\right)$.

\subsubsection{The poll}

In order to obtain assessments about the perception of fineness, $L$ subjects will be asked to assign images from $\mathcal{I}$ to classes, so that each class has associated a perception degree of fineness. In particular, $L=20$ subjects have participated in the poll and 9 classes have been considered (the nine images in Fig. 1 show the nine representative 
images for each class used in this poll). As result, a vector of 20 assessments $\Theta^{i}=\left[o_{1}^{i}, \ldots, o_{20}^{i}\right]$ is obtained for each image $I_{i} \in \mathcal{I}$. The degree $o_{j}^{i}$ associated to the assessment given by the subject $S_{j}$ to the image $I_{i}$ is computed as $o_{j}^{i}=(9-l) * 0.125$, where $l \in\{1, \ldots, 9\}$ is the index of the class $C_{l}$ to which the image is assigned by the subject.

\subsubsection{Assessment aggregation}

For each image in $\mathcal{I}$, one assessment $v^{i}$ that summarizes the $\Theta^{i}$ values is needed. To aggregate opinions, an OWA operator guided by a quantifier have been used. Concretely, the quantifier "the most" has been used, which allows to represent the opinion of the majority of the subjects [11].

\subsection{Fitting the membership function}

At this point, the aim is to obtain, for a given measure $P_{k} \in \mathcal{P}$, the corresponding type- 2 membership function $\tilde{\mathcal{T}}_{k}$. We will restrict ourselves to type-2 functions such that the membership function of the secondary fuzzy set $\tilde{\mathcal{T}}_{k, x}$ is a triangular function defined on $[0,1]$ with parameters $a_{x} \leq$ $b_{x} \leq c_{x} \in[0,1]$. Thus, $\tilde{\mathcal{T}}_{k}$ is defined in the following way:

$$
\tilde{\mathcal{T}}_{k}(x, u)= \begin{cases}0 & u \leq a_{x} \\ \frac{u-a_{x}}{b_{x}-a_{x}} & a_{x} \leq u \leq b_{x} \\ \frac{c_{x}-u}{c_{x}-b_{x}} & b_{x} \leq u \leq c_{x} \\ 0 & u \geq c_{x}\end{cases}
$$

The rationale behind this choice is the following: when there is no uncertainty about the fuzzy model, each value $x \in \mathbb{R}$ is assigned a membership degree in $[0,1]$ (type- 1 approach); hence, a natural way to take into account uncertainty about the model (type-2 approach) is to assume there is uncertainty about the membership degree, so that instead of a precise value $b_{x} \in[0,1]$, we will use an approximate value of the form around $b_{x}$, that is usually modeled as a triangular membership function with kernel $b_{x}$. If there is no uncertainty about the fuzzy model, we are in the case $a_{x}=b_{x}=c_{x}$, corresponding to a type-1 fuzzy set (in which case we denote the type- 1 membership function as $\mathcal{T}_{k}$, verifying $\mathcal{T}_{k}(x)=a_{x}=b_{x}=c_{x}$ ).

The function proposed in Eq. 2 can be characterized and described by means of three functions:

$$
f_{\tilde{\mathcal{T}}_{k}}^{a}, f_{\tilde{\mathcal{T}}_{k}}^{b}, f_{\tilde{\mathcal{T}}_{k}}^{c}: \mathbb{R} \rightarrow[0,1]
$$

with $f_{\tilde{\mathcal{T}}_{k}}^{a}(x) \leq f_{\tilde{\mathcal{T}}_{k}}^{b}(x) \leq f_{\tilde{\mathcal{T}}_{k}}^{c} \forall x \in \mathbb{R}$, so that, for each $(x, u) \in \mathbb{R} \times[0,1]$, the type-2 membership function $\tilde{\mathcal{T}}_{k}$ is calculated as in Eq.2 using as parameters $a_{x}=f_{\tilde{\mathcal{T}}_{k}}^{a}(x)$, $b_{x}=f_{\tilde{\mathcal{T}}_{k}}^{b}(x)$, and $c_{x}=f_{\tilde{\mathcal{T}}_{k}}^{c}(x)$. Fig. 2 shows graphically an example of this kind of type- 2 membership function. A bidimensional representation is also showed in Fig. 3.
Notice that an interval-valued fuzzy set (a special case of type-2 fuzzy set) can be obtained just considering $a_{x}=$ $f_{\tilde{\mathcal{T}}_{k}}^{a}(x)$ and $c_{x}=f_{\tilde{\mathcal{T}}_{k}}^{c}(x)$ as the extremes of the interval, that is, assigning values 1 to pairs $(x, u) \in \mathbb{R} \times[0,1]$ such that $f_{\tilde{\mathcal{T}}_{k}}^{a}(x) \leq u \leq f_{\tilde{\mathcal{T}}_{k}}^{c}(x)$, and 0 otherwise.

Our approach to obtain the membership function $\tilde{\mathcal{T}}_{k}$ is based on obtaining the functions $f_{\tilde{\mathcal{T}}_{k}}^{a}, f_{\tilde{\mathcal{T}}_{k}}^{b}$, and $f_{\tilde{\mathcal{T}}_{k}}^{c}$. Since we are searching for a function which associates the measure values $\left(m_{k}^{i, w}\right)$ and the human assessments of fineness $\left(v^{i}\right)$, we propose to estimate the three functions by fitting suitable curves on the basis of the multiset of points $\Psi_{k}=\left\{\left(m_{k}^{i, w}, v^{i}\right), i=1, \ldots, N ; w=1, \ldots, W\right\}$, with $N$ being the number of images and $W$ the number of windows considered for each image. In the next sections we shall introduce and justify the approaches followed to obtain $f_{\tilde{\mathcal{T}}_{k}}^{b}$ (section 2.2.1) and both $f_{\tilde{\mathcal{I}}_{k}}^{a}$ and $f_{\tilde{\mathcal{I}}_{k}}^{c}$ (section 2.2.2).

\subsubsection{Estimation of the function $f_{\tilde{\mathcal{T}}_{k}}^{b}$}

As we mentioned in the previous section, the secondary fuzzy set $\tilde{\mathcal{T}}_{k, x}$ represents the uncertainty about the actual membership degree of $x$ by means of a triangular fuzzy set whose semantics is "around $b_{x}$ ". The first step is to determine the most suitable value of $b_{x} \forall x \in \mathbb{R}$ that, as we explained, will be given by $b_{x}=f_{\tilde{\mathcal{T}}_{k}}^{b}(x)$. Our idea is that the value in $[0,1]$ with higher possibility to be $b_{x}$ can be obtained as a central tendency measure calculated in the subset of pairs in $\Psi_{k}$, so we shall calculate $f_{\tilde{\mathcal{T}}_{k}}^{b}$ as a function representing the central tendency of the set $\Psi_{k}$

Following this idea, we propose to define $f_{\tilde{\mathcal{T}}_{k}}^{b}$ as a function of the form ${ }^{3}$

$f_{\tilde{\mathcal{T}}_{k}}^{b}\left(x ; a_{n} \ldots a_{0}, \alpha, \beta\right)= \begin{cases}0 & x<\alpha, \\ \operatorname{poly}^{n}\left(x ; a_{n} \ldots a_{0}\right) & \alpha \leq x \leq \beta, \\ 1 & x>\beta\end{cases}$

with $\operatorname{poly}^{n}\left(x ; a_{n} \ldots a_{0}\right)$ being a polynomial function

$$
\operatorname{poly}^{n}\left(x ; a_{n} \ldots a_{0}\right)=a_{n} x^{n}+\ldots+a_{1} x^{1}+a_{0}
$$

In our proposal, the parameters $a_{n} \ldots a_{0}, \alpha$ and $\beta$ of the function $f_{\tilde{\mathcal{T}}_{k}}^{b}$ are calculated by carrying out a robust fitting on $\Psi_{k}$ with the constraint to obtain a monotonic function. For the polynomial function, the cases of $n=1,2,3$ (i.e. linear, quadratic and cubic functions) have been considered. In this paper, the robust fitting based on M-estimators (a generalization of the least squares fitting) has been used [12]. For each image $I_{i} \in \mathcal{I}, W=2000$ subimages of size $32 \times 32$ have been considered (so 16000 points have been used for the fitting).

\footnotetext{
${ }^{3}$ Note that this function is defined for measures that increase according to the perception of fineness. For those that decreases, the function needs to be changed appropriately
} 


\subsubsection{Estimation of the functions $f_{\tilde{\mathcal{T}}_{k}}^{a}$ and $f_{\tilde{\mathcal{T}}_{k}}^{c}$}

As we have seen, the triangular secondary fuzzy set $\tilde{\mathcal{T}}_{k, x}$ has the semantics "around $b_{x}$ ", with $b_{x}=f_{\tilde{\mathcal{T}}_{k}}^{b}(x)$ calculated as the central tendency of $\Psi_{k}$. Not only the value of this central tendency, but also the actual semantics of around must be calculated for each $x \in \mathbb{R}$. This semantics is given by the support of $\tilde{\mathcal{T}}_{k, x}$ (the interval $\left[a_{k}, c_{k}\right]$ ) and, in our view, should reflect the variability or deviation of the values $v_{i}$ around the expected central value $b_{x}$.

Thus, we propose to estimate the functions $f_{\tilde{\mathcal{T}}_{k}}^{a}$ and $f_{\tilde{\mathcal{T}}_{k}}^{c}$ as central tendencies of the variability above and below $b_{x}=f_{\tilde{\mathcal{T}}_{k}}^{b}(x)$, respectively. As in the previous section, we propose to obtain the two functions by fitting suitable curves on the basis of a set of points, noted as $\Upsilon_{k}^{a}$ and $\Upsilon_{k}^{c}$, representing the variability above and below $f_{\tilde{\mathcal{T}}_{k}}^{b}$, respectively. How we obtain these sets $\Upsilon_{k}^{a}$ and $\Upsilon_{k}^{c}$ is explained next.

For each point $\mathbf{p}_{k}^{i, w}=\left(m_{k}^{i, w}, v^{i}\right) \in \Psi_{k}$ we define the following deviation measure

$$
\sigma_{k}^{i, w, \Omega}=\sqrt{\frac{\sum_{\mathbf{p}_{k}^{i^{\prime}, w^{\prime}} \in \Omega}\left(v^{i^{\prime}}-f_{\tilde{\mathcal{T}}_{k}}^{b}\left(m_{k}^{i, w}\right)\right)^{2} w e_{k}^{i^{\prime}, w^{\prime}}}{\sum_{\Omega} w e_{k}^{i^{\prime}, w^{\prime}}}}
$$

with $\Omega$ being a set of points in $\Psi_{k}$ around $\mathbf{p}_{k}^{i, w}$, and $w e_{k}^{i, w}$ being the weight calculated for $\mathbf{p}_{k}^{i, w}$ during the $f_{\tilde{\mathcal{T}}_{k}}^{b}$ fitting procedure.

Let $\bar{\Omega}_{k}^{i, w}=\left\{\mathbf{p}_{k}^{i^{\prime}, w^{\prime}} \in \Psi_{k},\left\|\mathbf{p}_{k}^{i^{\prime}, w^{\prime}}-\mathbf{p}_{k}^{i, w}\right\| \leq \lambda ; v^{i^{\prime}} \geq\right.$ $\left.f_{\tilde{\mathcal{T}}_{k}}^{b}\left(m_{k}^{i^{\prime}, w^{\prime}}\right)\right\}$ be the set of points around $\mathbf{p}_{k}^{i, w}$ (at a distance lower than $\lambda$ ) located above the function $f_{\tilde{\mathcal{T}}_{k}}^{b}$. In a similar way, let $\underline{\Omega}_{k}^{i, w}=\left\{\mathbf{p}_{k}^{i^{\prime}, w^{\prime}} \in \Psi_{k},\left\|\mathbf{p}_{k}^{i^{\prime}, w^{\prime}}-\mathbf{p}_{k}^{i, w}\right\| \leq \lambda ; v^{i^{\prime}} \leq\right.$ $\left.f_{\tilde{\mathcal{T}}_{k}}^{b}\left(m_{k}^{i^{\prime}, w^{\prime}}\right)\right\}$ be the set of points around $\mathbf{p}_{k}^{i, w}$ and located below the function $f_{\tilde{\mathcal{T}}_{k}}^{b}$. In this paper, $\lambda$ has been selected to ensure that the size of both $\bar{\Omega}_{k}^{i, w}$ and $\underline{\Omega}_{k}^{i, w}$ is 30 in each case (size enough for statistical analysis).

Using the previous concepts, we define ${ }^{4} \Upsilon_{k}^{a}=$ $\left\{\left(m_{k}^{i, w}, \sigma_{k}^{i, w, \underline{\Omega}}\right), i=1, \ldots, N ; w=1, \ldots, W\right\}$ as the set of pairs representing the deviation above the function $f_{\tilde{\mathcal{I}}_{k}}^{b}$. In a similar way, let $\Upsilon_{k}^{c}=\left\{\left(m_{k}^{i, w}, \sigma_{k}^{i, w, \bar{\Omega}}\right), i=1, \ldots, N ; w=\right.$ $1, \ldots, W\}$ be the set of pairs representing the deviation below the function $f_{\tilde{\mathcal{T}}_{k}}^{b}$.

Finally, we propose to define $f_{\tilde{\mathcal{T}}_{k}}^{a}$ (resp. $f_{\tilde{\mathcal{T}}_{k}}^{c}$ ) following the Eq.4 and obtaining the parameters $a_{n} \ldots a_{0}, \alpha$ and $\beta$ by carrying out a robust fitting on $\Upsilon_{k}^{a}$ (resp. $\Upsilon_{k}^{c}$ ) in the same way we did for the $f_{\tilde{\mathcal{T}}_{k}}^{b}$ function (section 2.2.1).

\footnotetext{
${ }^{4}$ In order to simplify the notation, the indexes have been removed in $\bar{\Omega}_{k}^{i, w}$ and $\underline{\Omega}_{k}^{i, w}$
}

Table 1. Error related to each model and parameters for the measure of Amadasun

\begin{tabular}{|c|c|c|c|c|c|}
\hline Measure & Error & \multicolumn{4}{|c|}{ Parameters for Amadasun } \\
\hline Amadasun [1] & 0.1290 & & $f_{\tilde{\mathcal{T}}_{k}}^{b}$ & $f_{\tilde{\mathcal{T}}_{h}}^{a}$ & $f_{\tilde{\mathcal{T}}_{k}}^{c}$ \\
\hline Correlation [3] & 0.1353 & $\alpha$ & 0.1735 & 0.2089 & $\frac{{ }_{k}}{0.1482}$ \\
\hline Abbadeni [13] & 0.1564 & $\beta$ & 0.6425 & 0.8727 & 0.5512 \\
\hline FD [14] & 0.1769 & $a_{0}$ & 1.8767 & 1.9037 & 2.6750 \\
\hline Tamura [2] & 0.1806 & $a_{1}$ & -6.3436 & -6.1434 & -16.090 \\
\hline $\mathrm{ED}[15]$ & 0.1846 & $a_{2}$ & 8.2216 & 10.982 & 37.569 \\
\hline Weszka [16] & 0.1873 & $a_{3}$ & -4.5051 & -12.021 & -37.168 \\
\hline DGD [17] & 0.1955 & $a_{4}$ & 0.0000 & 5.3161 & 10.878 \\
\hline LH [3] & 0.2011 & & & & \\
\hline SRE [18] & 0.2108 & & & & \\
\hline SNE [19] & 0.2156 & & & & \\
\hline Newsam [20] & WSD & & & & \\
\hline Entropy [3] & WSD & & & & \\
\hline Uniformity[3] & WSD & & & & \\
\hline FMPS [21] & WSD & & & & \\
\hline Variance[3] & NR & & & & \\
\hline Contrast [3] & NR & & & & \\
\hline
\end{tabular}

\subsubsection{Goodness of the fitting}

To analyze the performance of the fitting, we propose the following goodness measure calculated as a weighted mean of absolute differences:

$E_{k}=\frac{\sum_{i, w}\left|f_{\tilde{\mathcal{T}}_{k}}^{b}\left(m_{k}^{i, w}\right)-v^{i}\right| \cdot\left(1-\tilde{\mathcal{T}}_{k}\left(m_{k}^{i, w}, v^{i}\right)\right) \cdot w e_{k}^{i, w}}{\sum_{i, w} w e_{k}^{i, w}}$

Table 1 shows the error obtained for each measure $P_{k} \in$ $\mathcal{P}$ sorted in increasing order. The parameter values of the measure with the lowest error are also shown in Table 1. It should be noticed that we haven't carried out the fitting with six of the measures. Four of them (marked with WSD) are rejected because their values are affected by the window size. The other two (marked with NR) produce a diffuse cloud of points $\Psi_{F}$, so they do not provide representative information.

\section{Results}

In this section, the type-2 fuzzy set $\tilde{\mathcal{T}}_{k}$ with the least error (obtained for Amadasun's measure and defined by the parameter values shown in Table 1) will be applied in order to analyze the performance of the proposed model. Fig. 2 shows graphically the type- 2 membership function $\tilde{\mathcal{T}}_{k}(x, u)$ used in our experiments.

Let's consider Fig. 3(A) corresponding to a mosaic made by several images, each one with a different increasing perception degree of fineness. Images in Fig.3(B,C) show, respectively, a mapping from the original image to the 


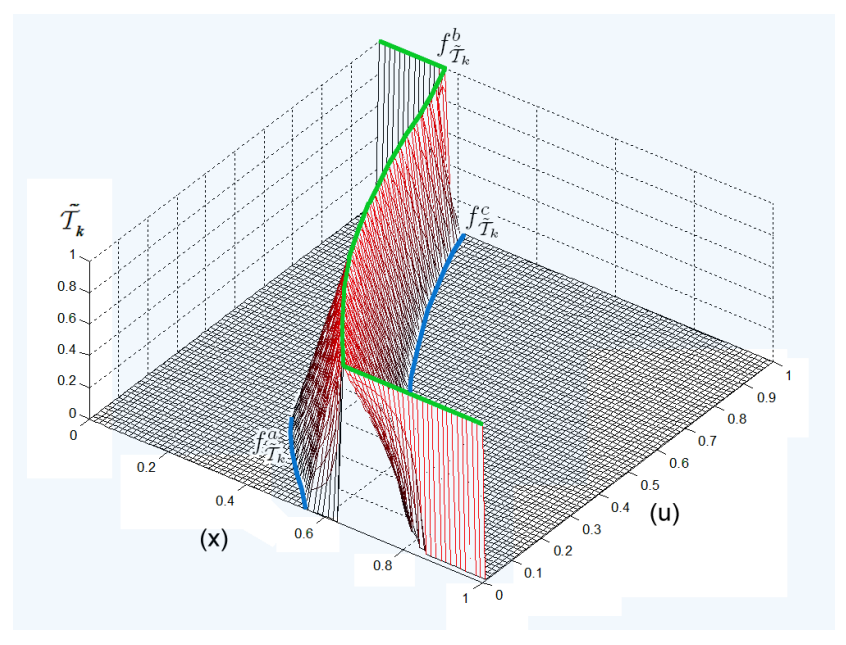

Figure 2. Type-2 membership function

kernel (given by $b_{x}$ ) and the support width (given by $\left.\left|a_{x}-c_{x}\right|\right)$ of the secondary fuzzy sets $\tilde{\mathcal{T}}_{k, x}$. For each pixel in the original image, a centered window of size $32 \times 32$ has been analyzed to obtain $a_{x}=f_{\tilde{\mathcal{T}}_{k}}^{a}(x), b_{x}=$ $f_{\tilde{\mathcal{T}}_{k}}^{b}(x)$ and $c_{x}=f_{\tilde{\mathcal{T}}_{k}}^{c}(x)$. Thus, Fig. 3(B) represents the degree in which the human perceives the texture, with a white grey level meaning maximum perception of fineness, and a black one meaning no perception of fineness (i.e., maximum perception of coarseness). Fig. $3(\mathrm{C})$ can be interpreted as the uncertainty in the estimated fineness membership degree, where a white grey level means maximum uncertainty, and a black one means no uncertainty. A bidimensional representation of the type- 2 membership function used in this experiment is showed in the top of Fig. 3 (a zenithal view of Fig.2). As example, this graph shows the mapping process for two different windows of the original image (marked as 1 and 2 in Fig.3(A)). It can be noticed that our model captures the evolution of the perception degrees of fineness, showing high (resp. low) degrees for fine (resp. coarse) textures. In addition, the uncertainty in the degrees is also captured (Fig.3(C)), showing less uncertainty in the very fine and very coarse cases than in the intermediate cases.

Fig. 4 presents an example where the proposed type- 2 fuzzy set has been employed for pattern recognition. In this case, the figure shows a microscopy image (Fig. 4(A)) corresponding to the microstructure of a metal sample. The lamellae indicates islands of eutectic, which are to be separated from the uniform light regions. The brightness values in regions of the original image are not distinct, so texture information is needed for extracting the uniform areas. This fact is shown in Fig. $4(\mathrm{~B} 1, \mathrm{~B} 2)$, where a thresholding on the original image is displayed (homogeneous regions cannot be separated from

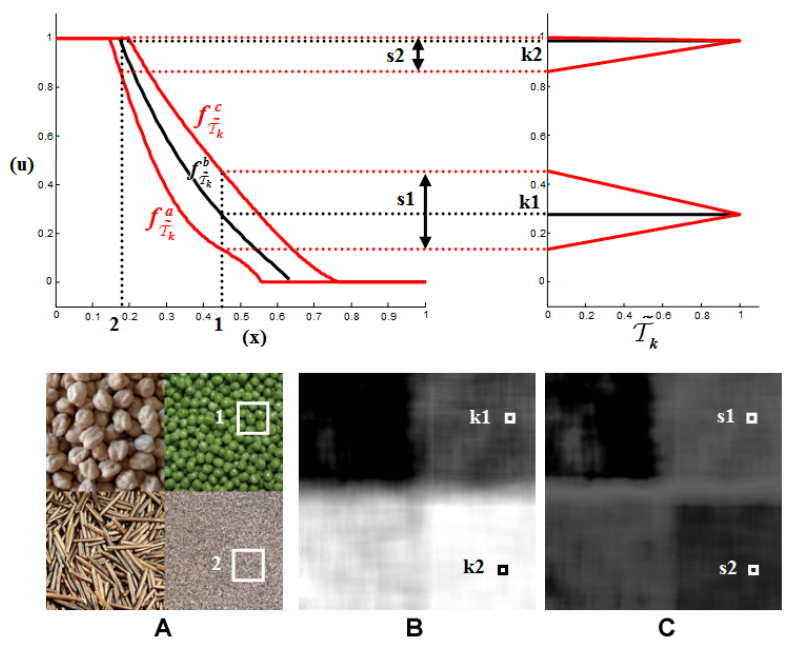

Figure 3. Mosaic image example (A) Original
image (B)(C) Mapping from the original image
to the kernel and support width of the
secondary fuzzy sets

the textured ones as they "share" brightness values). Fig. $4(\mathrm{C} 1, \mathrm{C} 2)$ shows a mapping from the original image to the kernel $\left(b_{x}\right)$ and the support width $\left(\left|a_{x}-c_{x}\right|\right)$ of the secondary fuzzy sets $\tilde{\mathcal{T}}_{k, x}$. Thus, Fig. 4(C1) represents the degree in which the human perceives the texture and Fig. 4(C2) the uncertainty of that degree. It can be noticed that uniform regions in Fig. 4(C1) correspond to areas with low degrees of fineness (i.e., high coarseness), so if only the pixels with fineness degree lower than 0.1 are selected (which it is equivalent to a coarseness degree upper than 0.9), the uniform light regions emerge with ease (Fig. 4(C3,C4)). In addition, Fig. 4(C2) shows low uncertainty in the degrees associated to these areas.

\section{Conclusions and future works}

In this paper, type- 2 fuzzy sets for coarseness-fineness representation have been defined, relating measures values (the reference set) with the human perception of this texture property. In order to obtain assessments about fineness perception, a group of human subjects has been polled. From the collected data, a fitting procedure has been applied in order to obtain the parameters defining the membership functions of the secondary fuzzy sets (in our case, triangular functions). The resulting type-2 fuzzy sets model both the imprecision in the interpretation of the measure value and the uncertainty about the coarseness degree associated to a measure value. In our study, the Amadasum's measure gives the best fitting; the results show a high connection between our model and the human perception of coarseness. 


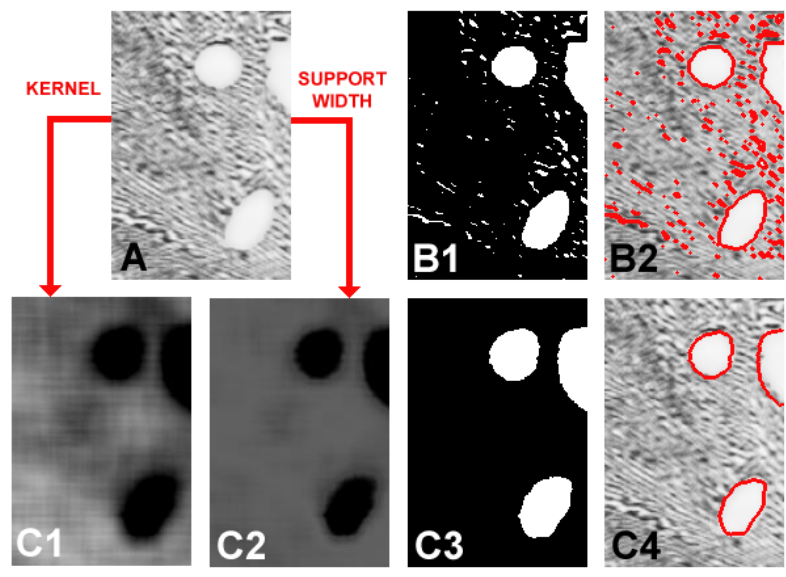

Figure 4. Pattern recognition example (A) Original image (B1) Binary image obtained by thresholding the original one (B2) Region outlines of $\mathrm{B} 1$ superimposed on original image (C1)(C2) Mapping from the original image to the kernel and support width of the secondary fuzzy sets (C3) Binary image obtained by thresholding C1 (C4) Region outlines of $\mathrm{C} 3$ superimposed on original image

As future work, the combination of several measures as reference set will be studied. In addition, the performance of the proposal will be analyzed in applications like textural classification or segmentation.

\section{References}

[1] M. Amadasun and R. King. Textural features corresponding to textural properties. IEEE Transactions on Systems, Man and Cybernetics, 19(5):1264-1274, 1989.

[2] H. Tamura, S. Mori, and T. Yamawaki. Textural features corresponding to visual perception. IEEE Transactions on Systems, Man and Cybernetics, 8:460-473, 1978.

[3] R.M. Haralick. Statistical and structural approaches to texture. Proceedings IEEE, 67(5):786-804, 1979.

[4] A. Barcelo, E. Montseny, and P. Sobrevilla. Fuzzy texture unit and fuzzy texture spectrum for texture characterization. Fuzzy Sets and Systems, 158:239-252, 2007.

[5] H.C. Lin, C.Y. Chiu, and S.N. Yang. Finding textures by textual descriptions, visual examples, and relevance feedbacks. Pattern Recognition Letters, 24(14):2255-2267, 2003.

[6] P. Puvanathasan and K. Bizheva. Interval type-ii fuzzy anisotropic diffusion algorithm for speckle noise reduction in optical coherence tomography images. Optics express, 17(2):733-746, 2009.
[7] H. Bustince, E. Barrenechea, M. Pagola, and R. Orduna. Construction of interval type 2 fuzzy images to represent images in grayscale. false edges. In Proc. of IEEE Fuzzy Systems Conference, pages 73-78, 2007.

[8] H.R. Tizhoosh. Image thresholding using type ii fuzzy sets. Pattern Recognition, 38(12):151-156, 2007.

[9] S. Tehami, A. Bigand, and O. Colot. Color image segmentation based on type-2 fuzzy sets and region merging. In ACIVS07, pages 943-954, 2007.

[10] J. Chamorro-Martínez, E. Galán-Perales, J.M. Soto-Hidalgo, and B. Prados-Suarez. Using fuzzy sets for coarseness representation in texture images. In Proceedings IFSA'07, pages 783-792, 2007.

[11] R.R. Yager. On ordered weighted averaging aggregation operators in multicriteria decisionmaking. IEEE Transactions on Systems, Man and Cybernetics, 18(1):183-190, 1988.

[12] A.E. Beaton and J.W. Tukey. The fitting of power series, meaning polynomials, illustrated on band-spectroscopic data. Technometrics, 16:147-185, 1974.

[13] N. Abbadeni, N. Ziou, and D.S. Wang. Autocovariance-based perceptual textural features corresponding to human visual perception. In Proc. of 15th International Conference on Pattern Recognition, volume 3, pages 901-904, 2000.

[14] S. Peleg, J. Naor, R. Hartley, and D. Avnir. Multiple resolution texture analysis and classification. IEEE Transactions on Pattern Analysis and Machine Intelligence, (4):518-523, 1984.

[15] J. Canny. A computational approach to edge detection. IEEE Transactions on Pattern Analysis and Machine Intelligence, 8(6):679-698, 1986.

[16] J.S. Weszka, C.R. Dyer, and A. Rosenfeld. A comparative study of texture measures for terrain classification. IEEE Transactions on Systems, Man and Cybernetics, 6:269-285, 1976.

[17] S.I. Kim, K.C. Choi, and D.S. Lee. Texture classification using run difference matrix. In Proc. of IEEE 1991 Ultrasonics Symposium, volume 2, pages 1097-1100, December 1991.

[18] M.M. Galloway. Texture analysis using gray level run lengths. Computer Graphics and Image Processing, 4:172-179, 1975.

[19] C. Sun and W.G. Wee. Neighboring gray level dependence matrix for texture classification. Computer Vision, Graphics and Image Processing, 23:341-352, 1983.

[20] S.D. Newsam and C. Kammath. Retrieval using texture features in high resolution multi-spectral satellite imagery. In Data Mining and Knowledge Discovery: Theory, Tools, and Technology VI, SPIE Defense and Security, April 2004.

[21] H. Yoshida, D.D. Casalino, B. Keserci, A. Coskun, O. Ozturk, and A. Savranlar. Wavelet-packet-based texture analysis for differentiation between benign and malignant liver tumours in ultrasound images. Physics in Medicine and Biology, 48:3735-3753, 2003. 